\title{
Particle Methods for Nonequilibrium Hypersonic and Plasma Flows
}

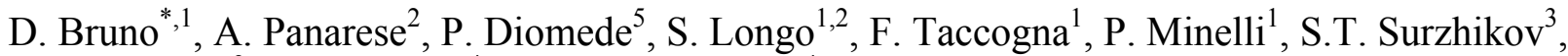 \\ A.S. Dikaljuk ${ }^{3}$, P. De Palma ${ }^{4}$ and M.D. de Tullio ${ }^{4}$
}

\author{
${ }^{I}$ Istituto di Metodologie Inorganiche e dei Plasmi, Consiglio Nazionale delle Ricerche, via G. Amendola 122/D, 70126 \\ Bari, Italy \\ ${ }^{2}$ Department of Chemistry, University of Bari, via E. Orabona 4, 70126 Bari, Italy \\ ${ }^{3}$ Institute for Problems in Mechanics, Russian Academy of Sciences, Pr. Vernadskogo 101-1, 119526 Moscow, Russia \\ ${ }^{4}$ Dipartimento di Meccanica, Matematica e Management \& Centro di Eccellenza in Meccanica Computazionale, \\ Politecnico di Bari, via Re David, 200, 70125 Bari, Italy \\ ${ }^{5}$ Plasma Processing Laboratory, Department of Chemical and Biomolecular Engineering, University of Houston, \\ Houston, TX 77204-4004, USA
}

\begin{abstract}
Kinetic modelling of nonequilibrium flows is described as it applies to hypersonic phenomenology. A Monte Carlo method is described for the study of species separation on shock wave fronts; a Particle in Cell with Monte Carlo Collisions (PIC/MCC) technique is described for the simulation of dust in plasma flows; modelling of nonequilibrium radiation in shock heated gases; implementation of slip models for the description of separation zones occurring in shockboudary layer interactions.
\end{abstract}

Keywords: Monte Carlo, nonequilibrium plasma, PIC, shock wave.

\section{INTRODUCTION}

The study of high-speed reacting gas flows, as it arises in aerospace applications, involve the consideration of nonequilibrium effects. Current research on nonequilibrium modelling in Computational Fluid Dynamics (CFD) solvers has progressed substantially in including many nonequilibrium effects in the flow description. Still, there are flow conditions where the degree of nonequilibrium is such that the hydrodynamic description fails and kinetic methods are required for the correct description of the flowfield. In this cases, new methods must be used to solve the governing equations at the kinetic level. In this study we report on a number of situations where kinetic nonequilibrium effects have a large impact on the overall flowfield and we introduce the kinetic models used to tackle them.

In section 2, the problem of diffusion in shock wave fronts in analyzed. Here, large temperature and density gradients prevent the Navier-Stokes description from being accurate. A particle (i.e. Monte Carlo) method is devised to solve the relevant Boltzmann transport equations both for the bulk gas and for a trace species. It is shown, in particular, how the proposed method can successfully describe the transport of the seed species to any desired level of accuracy, thus overcoming a major limitation of standard Monte Carlo

*Address correspondence to this author at the Istituto di Metodologie Inorganiche e dei Plasmi, Consiglio Nazionale delle Ricerche, via G. Amendola 122/D, 70126 Bari, Italy; Tel: +390805929510;

Fax:+390805929520; E-mail: domenico.bruno@ba.imip.cnr.it methods for flow simulation (like, e.g. Direct Simulation Monte Carlo, DSMC).

In section 3, a Particle-in-Cell (PIC) technique is described for the simulation of dust grains inside a plasma. This is relevant for the study of dust grains effects in complex plasmas, but it is also interesting for aerospace applications in that it shows how charged particle kinetic effects affect the boundary layer of macroscopic objects flying through a partially ionised gas.

In section 4, a theoretical framework is presented for the simulation of the nonequilibrium radiation emitted by shock heated gas mixtures. While the gas flowfield is here described by hydrodynamic equations, with due account of thermal and chemical nonequilibrium effects, a detailed kinetic framework is developed for the description of the radiation field. This is required when comparing simulation results to detailed experimental spectral measurements.

Finally, in section 5 a approach is proposed for the description of separation zones in CFD simulations. The Immersed Boundary (IB) method has been shown to be a promising technique to include boundary conditions in CFD solvers without requiring a body-fitted mesh, thus providing a significant gain in flexibility and computational requirements. In separation zones, however, rarefied gas effects make their appearance and standard boundary conditions fail to describe the flow accurately. In this study we show that rarefied gas effects (velocity slip and temperature jump) can effectively be described in a IB framework. Predictions of the model are assessed by comparison to accurate DSMC simulations for a hypersonic ramp flow in Nitrogen. 


\section{NEUTRAL SEPARATION IN SHOCK WAVES}

\subsection{Monte Carlo Model}

Results of this sections have been obtained by using a Monte Carlo (MC) method in a "two stage" calculation based on the method described in [1] and shortly reviewed here, and besides, a discussion of a test case is provided where separation effects along specific sections of a $2 \mathrm{D}$ shock produced by a blunt body are discussed for the first time. In a first stage the bulk flow field is established by solving the main component, so that, in a second stage, it is possible to handle both the components which are separately solved one after the other.

The computational module implementing the first stage solves the nonlinear transport equation

$$
\frac{\partial f_{b}}{\partial t}+\mathbf{v} \cdot \frac{\partial f_{b}}{\partial \mathbf{r}}=\int d \Omega d^{3} v_{1} g \sigma_{b}(g, \vartheta)\left(f_{b}^{\prime} f_{M}^{\prime}\left(v_{1}^{\prime}\right)-f_{b} f_{M}\left(v_{1}\right)\right) \text {. }
$$

Here $\sigma_{b}(g, \vartheta)$ is the differential cross section (where $g$ is the relative speed), and $f_{b}$ the translational distribution of the bulk, whereas $f_{M}$ is the corresponding local equilibrium distribution based on local macroscopic variables while $f_{M}(\mathbf{r}, \mathbf{v})$ is determined from the simulation making the whole process nonlinear.

The Test Particle Monte Carlo (TPMC) method is employed to solve the transport equation in the nonlinear version explained in [2], in which a "virtual" target particle is sampled from a local equilibrium distribution to treat the particle collisions. Here the particles are handled and storaged in a way that is more similar to (BKG/MC) [3-5] than it is to Direct Simulation Monte Carlo (DSMC). For simplicity a fixed number of particles is used, a particle which leaves the simulation domain is injected back from the inlet. During a time step $\Delta t$, particles are moved according to their velocity and solid obstacles (aerodynamic control surfaces, thermal protection) are included in the model by marking as "filled" the corresponding mesh elements [6,7]. Each particle in the course of the nonlinear process contributes to the local average of a generic quantity $a$ :

$$
\langle a\rangle_{c}=\frac{\sum_{p} F_{c}\left(\mathbf{r}_{p}\right) a_{p}}{\sum_{p} F_{c}\left(\mathbf{r}_{p}\right)},
$$

where $c$ is the cell index, $p$ is the particle index, and $F_{c}(r)$ is a function that characterizes the $c$-th cell. This applies to the average number density, local temperature, etc. Every particle interacts with a virtual target particle by means of binary collisions, and, if the local number density of the target species at the time $t$ is denoted by $n(\mathbf{r}, t)$, the collision probability is

$$
P_{\mathrm{vv}_{1}}=1-\exp (-g \sigma(g) n(\mathbf{r}, t) \Delta t) \text {. }
$$

$\mathbf{v}, \mathbf{v}_{1}$, and $g=\left|\mathbf{r}-\mathbf{r}_{1}\right|$ are the particle velocity, the virtual particle velocity, and the relative speed, respectively, while $\sigma$ is the scattering cross section.

$f_{M}\left(\mathbf{r}, \mathbf{v}_{1}, t\right)$ is the distribution which describes the stochastic variable $\mathbf{v}, v_{1 i}$, the i-th Cartesian component of $\mathbf{v}_{1}$, can be calculated as follows. First a random number $\xi$ is generated from an ensemble with a $\exp (-\xi)$ distribution, which is in turn produced by means of the von Neumann rejection method. Secondly the averages $\left\langle v_{i}\right\rangle$, and $\left\langle(\mathbf{v}-\langle v\rangle)^{2}\right\rangle$ are computed in agreement with Eq. (2) Finally $v_{1 i}$ is obtained as

$v_{1 i}=\sqrt{\frac{2}{3} \xi\left\langle(\mathbf{v}-\langle v\rangle)^{2}\right\rangle}+\left\langle v_{i}\right\rangle$.

Let $\eta$ be a random number uniformly distributed between 0 and 1 , then the collision occurs if $P_{\mathbf{v v}_{1}}>\eta$. After the collision, a new relative velocity $\mathbf{g}^{\prime}$ is chosen at random by sampling it from a suitable distribution, and the particle velocity $\mathbf{v}^{\prime}$ is calculated as

$\mathbf{v}^{\prime}=\frac{m \mathbf{v}+m_{1} \mathbf{v}_{1}}{m+m_{1}}+\frac{m_{1}}{m+m_{1}} \mathbf{g}^{\prime}$

If the momentum transfer cross section is used, the isotropic distribution is the appropriate one.

The energy and momentum conservation is enforced by performing a new sampling, but the memory of the results of the previous sampling is kept, as the velocity of each particle is scaled to find an agreement with these results. For this purpose, the averages $\langle\mathbf{v}\rangle,\left\langle\mathbf{v}^{2}\right\rangle$ and the same quantities after the new sampling, i.e. $\left\langle\mathbf{v}^{\prime}\right\rangle$ and $\left\langle\mathbf{v}^{2}\right\rangle$, are calculated cell by cell, and finally the particle velocities are computed again, according to the formula

$\mathbf{v} \leftarrow\left(\mathbf{v}-\left\langle\mathbf{v}^{\prime}\right\rangle\right) \sqrt{\frac{\left\langle\mathbf{v}^{2}\right\rangle-a^{2}}{\left\langle\mathbf{v}^{\prime 2}\right\rangle-a^{\prime 2}}}+\langle\mathbf{v}\rangle$.

The developed model does not need a dynamic list of particles, as does the DSMC model, because each particle communicates with other particles only indirectly by means of the cell.

The cycle composed by the procedures of the first stage is iterated a sufficient number of times to reach the steady state, at which the quantities that characterize the main species and that define the flow field are calculated. These quantities, i.e. temperature, space resolved number density, and velocity components, are written in some output files, which are read from the code that implements the second 
stage and calculates the separation effects. The transport equation proposed is not strictly the Boltzmann equation, but a nonlinear equation obtained generalizing the linear transport method and requires the local energy conservation to be enforced. The approach proposed presents elements of novelty. As such, its accuracy is to be verified: such check has been performed in [1] by comparing the flow field calculated with this approach for a transition regime flow on a cylinder with a finite element solution of the Boltzmann equation, obtaining very close results.

In the second stage, TPMC method has been used to solve the following linear transport equation for $i$-th component:

$\frac{\partial f_{i}}{\partial t}+\mathbf{v} \cdot \frac{\partial f_{i}}{\partial \mathbf{r}}=\int d \Omega d^{3} v_{1} g \sigma_{i}(g, \vartheta)\left(f_{i}^{\prime} f_{M}^{\prime}\left(v_{1}^{\prime}\right)-f_{i} f_{M}\left(v_{1}\right)\right)$,

which has been obtained by setting the target distribution $f_{M}$ to the solution of of Eq. (1). Moreover $f_{i}$ is the translational distribution of the $i$-th species, while $\sigma_{i}$ is the differential cross section for the elastic scattering between the particles of the $i$-th component and the main species particles.

In this way the final quantities of both the main species and the impurity are determined by mean of a linear test particle method. If $T(\mathbf{r})$ and $n(\mathbf{r})$ are the local temperature and number density of the target species, the collision frequency, preliminarily estimated, is

$v_{i}(\mathbf{r})=C \cdot n(\mathbf{r}) \sigma_{i}(k T(\mathbf{r})) \sqrt{\frac{k T(\mathbf{r})}{\pi \mu_{i}}}$,

where $\mu_{i}=m_{i} m_{a} /\left(m_{i}+m_{a}\right)$ is the reduced mass. In the rigid sphere model, the temperature corrected average cross section, $\sigma(k T(\mathbf{r}))$, is a constant. $C$ is a proportionality constant, whose global value, which can be selected following the rule described in [1], is usually $\sim 10$. The random free time between two collisions is calculated by the usual relation

$t_{c}=-\frac{\ln \eta}{v_{i}(\mathbf{r})}$

where the random number $\eta$ is uniformly distributed between 0 and 1. Excess collisions are removed using the null collision method [1]. We deal with the boundary conditions, with the particle motion for the stochastic time $t_{c}$, and finally with the sampling of a main component atom as candidate partner for the next collision as we do in the first stage. A collision is effective only if the collision probability $P_{\mathrm{vv}_{1}}=n(\mathbf{r}) \sigma_{i}(g) g / v_{i}(\mathbf{r})$ is greater than a random fraction sampled from a uniform distribution. In this case Eq. (5) is used to calculate the post collision velocity.

In the second stage the grid is set up only to treat obstacles and to store values of the quantities calculated in the first stage as well as their functions, so that the information is not propagated along the grid and the stage itself is not affected by numerical diffusion.

\subsection{Neutral Separation}

The method developed has been applied to a seeded transonic gas flow where $\mathrm{He}$ is the main component and $\mathrm{Ar}$ is the seed. As the mass ratio of the components is rather high $\left(m_{A r} / m_{H e} \sim 10\right)$, the separation phenomena are distinct. As regards the choice of cross sections, for technical applications it is better to use those calculated by means of Variable Soft Sphere (VSS) model [8] or quantum calculations. Nevertheless, our goal is to compare the test case results with next results obtained by other models, so we have calculated the hydrodynamic quantities by using the rigid sphere model. For $\mathrm{He} / \mathrm{He}$ and $\mathrm{He} / \mathrm{Ar}$ scattering, we have employed the momentum transfer total cross sections reported in [9]. Different cross sections are used to study an expanding atomic beam, and the results obtained by a preliminary version of the code are presented in [1].

We have considered a flow impinging on a cylindrical obstacle with a surface which is an adiabatic diffuser. In the unperturbed region far from the obstacle the gas flows with a Mach number and a Knudsen number equal respectively to $M=3$ and $K n_{\infty}=\lambda_{\infty} / R=0.5$. Equating the physical and numerical particle fluxes, we obtain the coefficient $w$ by the formula:

$w=n_{0} M \frac{\sqrt{\gamma k T_{0} / m}}{\Phi_{p}}$,

which is valid in the case of supersonic injection with $M \geq 3$. Here $\gamma=5 / 3$ is the specific heat ratio and $\Phi_{p}$ is the flux of injected numerical particles, which is calculated by a time average during the simulation. In both stages the particles are $2 \times 10^{4}$. The grid of the simulation box consists of $200 \times 150$ cells and is uniform. In the first stage we need 4000 time steps before the steady state is reached. From this moment on, for the next 4000 time step, the dynamic quantities are sampled

\subsection{Results}

The results obtained for the test case are shown in the following figures.

Fig. (1) is the result for the main component (He) calculated by the nonlinear test particle method. In particular Fig. (1a) is the number density, Fig. (1b) is the temperature, Fig. (1c) is the $x$ component of the velocity and Fig. (1d) is the Mach number. These results which have the function to establish a bulk flow field have been calculated by assuming rigid sphere scattering. The results for the density of $\mathrm{He}$ are in good agreement with those obtained by deterministic numerical solution of the Boltzmann equation for an atomic gas in the same conditions and presented in [10]. The shock structure is clearly visible and it is better outlined by the side plots which reports the corresponding quantites along the white horizontal and vertical lines shown in the color plot. 

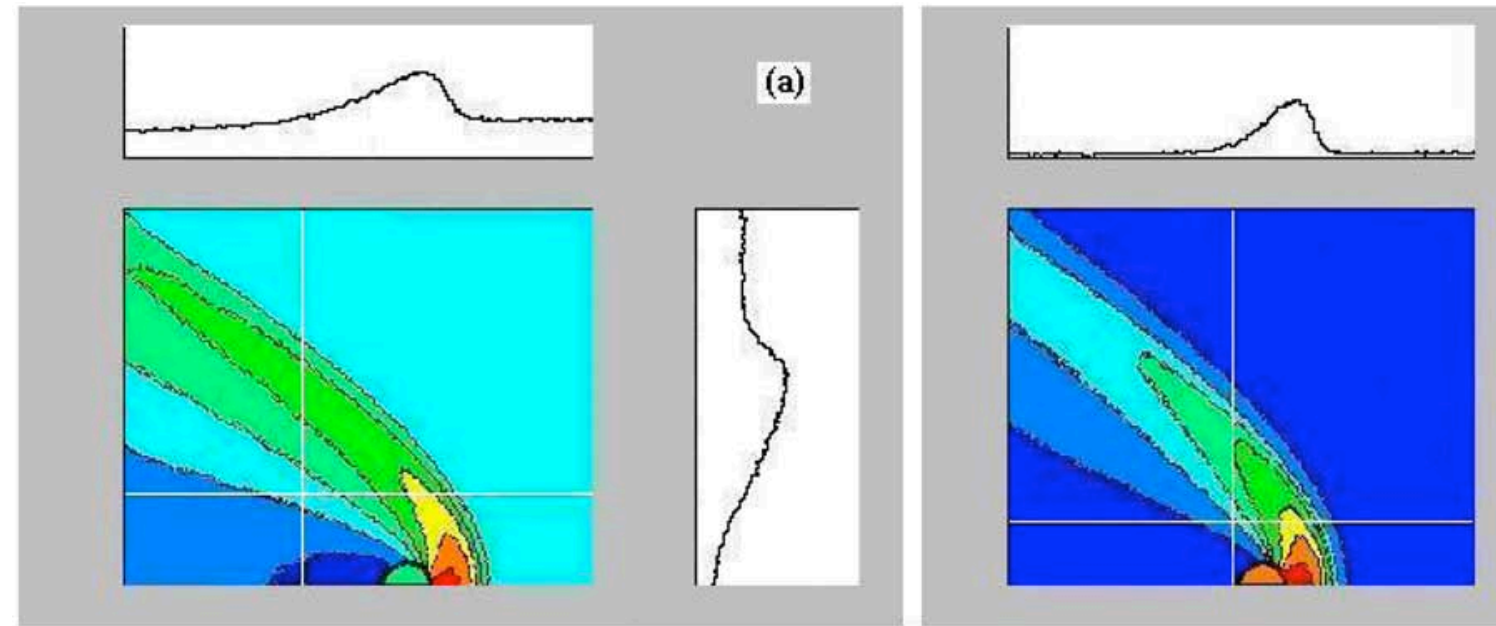

(b)
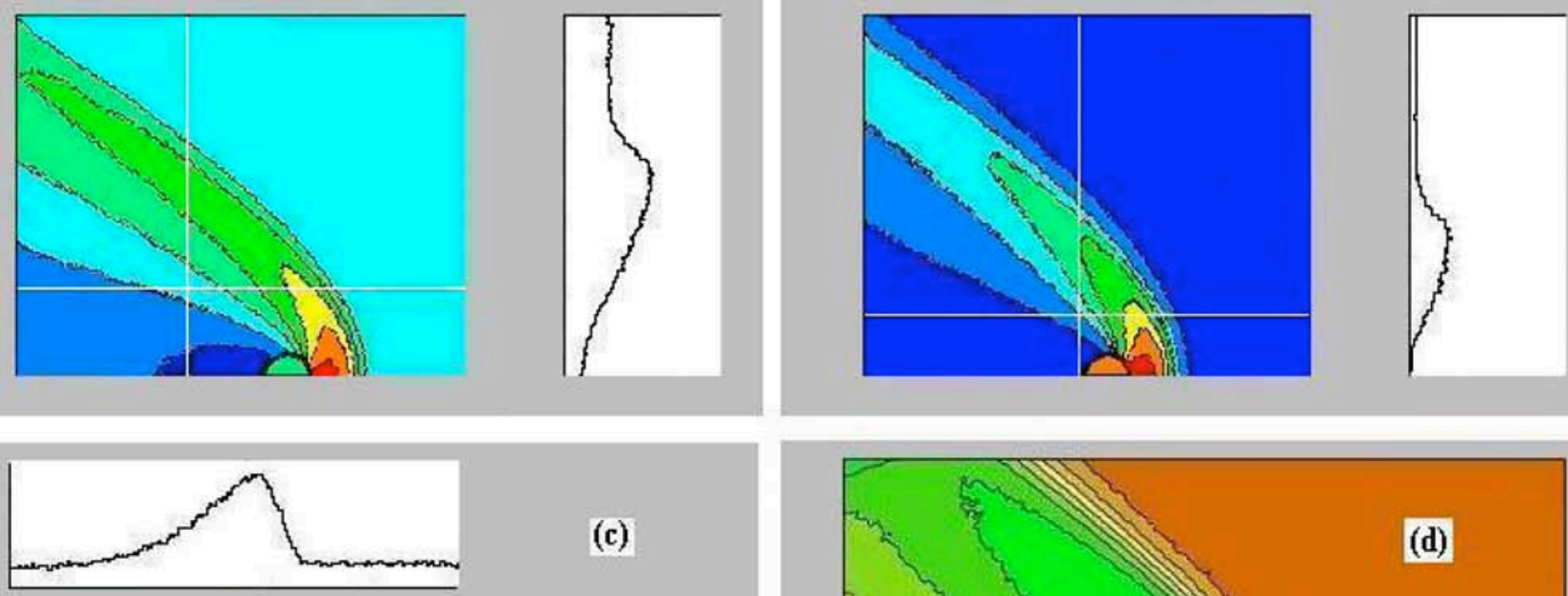

(c)
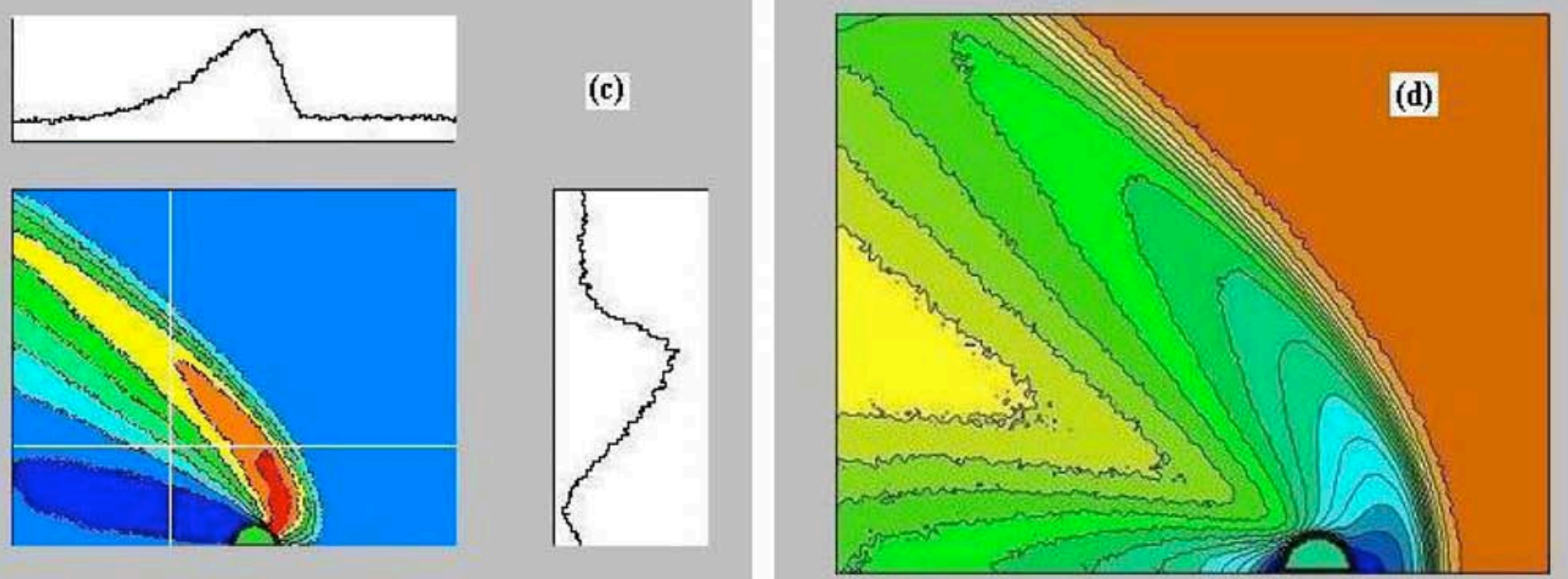

Fig. (1). Bulk flow field results calculated by the nonlinear TPMC method and by rigid sphere scattering. (a): number density. (b): temperature. (c): $x$ component of the velocity. (d): Mach number.
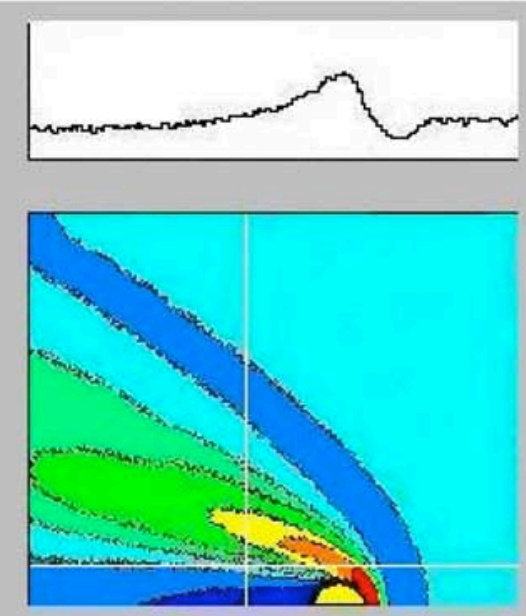

(a)

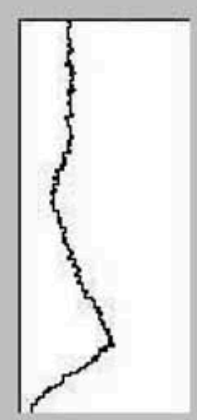

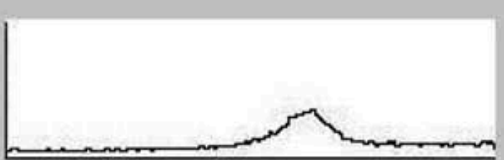

(b)

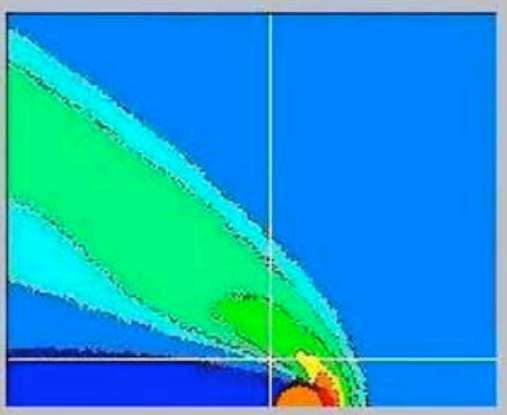

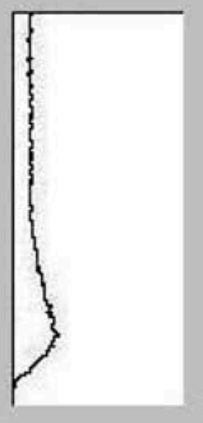

Fig. (2). Impurity component results calculated by the linear TPMC method and by rigid sphere scattering. (a): ratio of the impurity particle density with respect to the bulk one. (b): impurity particle density.

The next figures show results related to the impurity component which is calculated by the linear test particle. In particular Fig. (2a) report the ratio of the impurity particle density with respect to the bulk one, whereas Fig. (2b) report the impurity particle density.
The distributions represented in Figs. $(\mathbf{1}, \mathbf{2})$ are in agreement with expected theoretical distribution [11]. He atoms, which are less massive than $\mathrm{Ar}$ atoms, tend to concentrate entering the shock front, while are diluted just after the shock. In the wake there is another separation region, in fact the flow is enriched in He. 
The computational cost is about 14 minutes on a 3.6 $\mathrm{GHz}$ Intel Xeon processor, most of the time spent after the steady state is reached, in order to collect a satisfactory statistics and to achieve a desired level of variance.

\subsection{Test Case Conclusion}

In this section we have shown how the problem of component separation in transitional flows can be conveniently handled using a combination of Monte Carlo methods. In particular, the use of TPMC methods for the calculation of the detailed components in the framework of the previously established flow field is very efficient since such methods can provide an exact solution of the linear transport equation, thereby eliminating any numerical diffusion. In this way the separation effects can be studied even under conditions when they are relatively small.

Results for a 2D detached shock around a cylindrical body show an enrichment of the heavier component just after the shock, coherently with theoretical expectations based on kinetic theory [11]. Future extensions of the approach used in this paper will allow the determination of separation effects with molecular and ionic species taking into account the internal energy for the first case and the space charge effects for the second case.

\section{ABSORBING DUST IN STATIONARY PLASMA}

\subsection{Introduction}

The interaction of an object with the sorrounding plasma is still an open issue in plasma physics [12-15]. In particular, the particle charging and shielding is an unsolved problem in complex plasmas. The most used approach is the Orbital Motion Limited (OML) theory [16,17]. It claims that: (a) the particle surface potential is independent from the particle size and gas pressure; (b) the dust charge $Q_{d}$ is linearly dependent from the dust radius $r_{d}$; (c) the dust charge is independent from gas pressures $P$. The most accepted electric potential distribution around the particle is the Debye-Hückel (DH) form:

$\varphi_{D H}(r)=\varphi_{d} \frac{r_{d}}{r} e^{-\left(r-r_{d}\right) / \lambda}$

where $\lambda=\lambda_{D e} \lambda_{D i} / \sqrt{\lambda_{D e}^{-2}+\lambda_{D i}^{-2}}$ is the linearized Debye length.

In this work, we study an isolated absorbing spherical particle immersed in stationary, isotropic plasma with the effect of ion-neutral collisions for different particle radius and gas pressures.

\subsection{Numerical Model}

We have implemented a one-dimensional Particle-in-Cell (PIC) code [18-23]. Electrons and ions are tracked in the radial domain (motion in a central field of forces) using the electric field generating from their dynamics. The radial domain simulated $(\approx 40 \lambda)$ goes from the grain surface to the unperturbed neutral plasma. The operative conditions used are those typical of Argon $(\mu=39.95 \times 1836)$ gas discharge: plasma density $n_{p}=6 \cdot 10^{15} \mathrm{~m}^{-3}$; electron/ion temperature
$T_{e}=1 \mathrm{eV}$ and $T_{i}=0.2 \mathrm{eV} \quad(\tau=5)$; grain radius $r_{d}=0.8-200 \mu \mathrm{m}$, corresponding to $\rho_{d}=r_{d} / \lambda=0.02-5$; gas pressure $P=0-1000 P a$, corresponding to a Knudsen number $K n=5 \cdot 10^{-3}-\infty ; \quad$ gas temperature $T_{g}=1000 K$. The equations of motion are integrated through the leapfrog technique [19], while the particles plasma charge is deposited on the grid points taking into account the spherical metrics [24]. Poisson equation with the following boundary conditions:

$\left.\frac{d \varphi}{d r}\right|_{r_{d}}=-\frac{Q_{d}}{4 \pi \varepsilon_{0} r_{d}^{2}}$

$\left.\varphi\right|_{r_{\infty}}=0$

is solved by using the Thomas tridiagonal algorithm [25]. Ion-atom momentum MT and charge CT transfers collisions are processed through a detailed Test-Particle Monte Carlo technique [26].

\subsection{Results and Discussion}

\subsubsection{Ionic Flux, Dust Charge and Surface Potential}

Fig. (3) shows the ion flux $j_{i}=I_{i} / q$ collected on the particle surface as a function of the gas pressure for different particle radius. Ion-neutral collisions start to affect the ion dynamics already at few Pa. For larger pressure, more ions reach the dust surface before the ion current collected dramatically decreases. This behavior can be explained as follows: ion-neutral collisions induce first a destruction of ion orbital motion (producing in this way an increase of the ion current collected by the particle) and then, when the number of collisions become very large (collision-dominated regime), they lead to a drastic reduction of ion flux collected

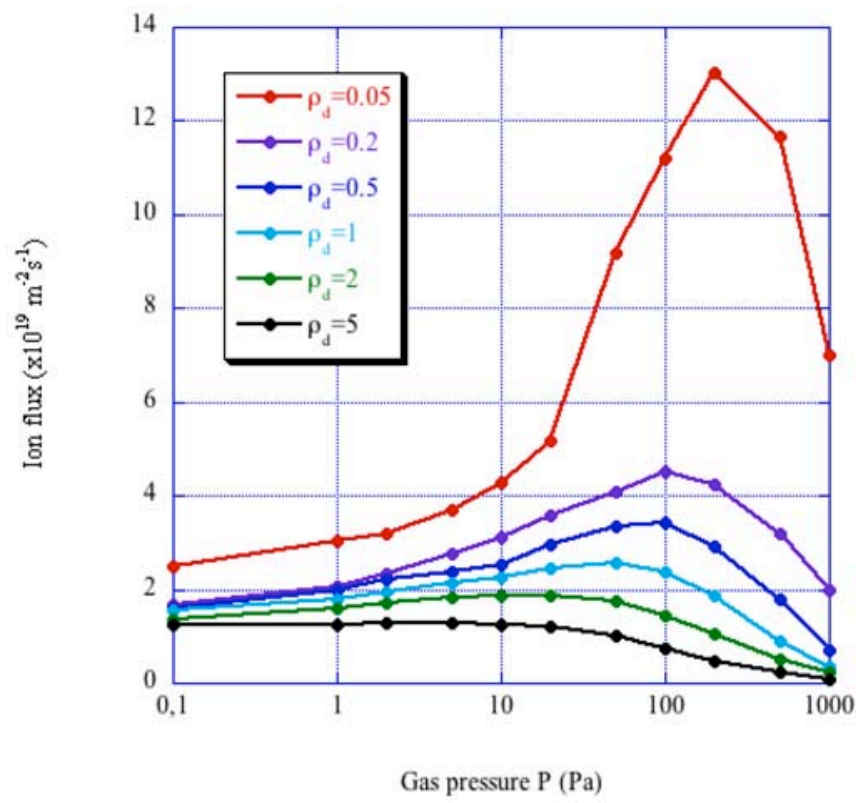

Fig. (3). Ion collection flux to the particle as a function of the gas pressure for different particle size. 
on the grain surface. For $\rho_{d}<5$ (the particle radius is smaller than the screening length), ion-neutral collisions enhance the current collected; this is the sign of orbital motion destruction. On the contrary, for $\rho_{d}>5$, the ion flux collected on the dust surface becomes a monotonic decreasing function of the gas pressure (thin sheath limit).

Fig. (4a) shows the surface potential $\varphi_{d}$ as a function of gas pressure for different particle size. The same behaviour of the ion current has been recovered: the surface potential reaches a peak, which is absent for large particles. Fig. (4b) shows the surface dust potential as a function of the dust radius for different collisional regimes. The dependence of surface potential from the particle size becomes more strong at high pressure (OML theory predicts no-dependence at all). In particular, for $P<10 \mathrm{~Pa}$ (weak collisional regime) the size-dependence is evident only for small dust $\left(\rho_{d}<0.5\right)$, while for intermediate collisional regimes the surface potential becomes strongly dependent from the particle size. This result is also a sign of the orbital motion destruction around the dust. Fig. (4c, d) show the dependance of dust particle charge from gas pressure at different particle size and from dust radius at different gas pressure, respectively. As the surface potential, the particle charge follows the ion flux behaviour too; in the transitional collision regime, the ion flow increase with the pressure results in a particle charge reduction, while the ion flow decrease for high collisional regime results in a particle charge increase.

\subsubsection{Ionic flux, Dust Charge and Surface Potential}

Fig. (5a) shows the radial profiles of electric potential around a particle of radius $\rho_{d}=1$ at three different pressures. It is evident that:

1. The far asymptote power law decay has been confirmed: $\varphi \propto r^{-2}$ in the collisionless case (dashed violet line), while $\varphi \propto r^{-1}$ in the strong collisional case (dashed light blue line);

2. The electric potential can be well fitted with a Yukawa form (11) with an effective screening length $\lambda_{\text {eff }}$ depending on the dust radius: for $\rho_{d}<<1, \lambda_{\text {eff }}$ is close to the ion Debye radius $\lambda_{D i}$, while for $\rho_{d}>>1, \lambda_{\text {eff }}$ increases reaching several $\lambda_{D e}$.

In Fig. (5b) the radial profile of the electric potential for $P=100 \mathrm{~Pa}$ at different particle radius have been reported. For small particles $\left(\rho_{d}<0.4\right)$, at the intermediate collisional regime ( $K n \approx 0.1$ ), the screening length is reduced due the reduction of the grain charge that is induced by an increased ion flux to the dust surface. While for large particles the screening length monotonically increases with $P$.

In Fig. (5c) radial profiles of electron (red lines) and ion (black lines) density for two different particle size $\left(\rho_{d}=0.5\right.$ and $\rho_{d}=5$ ) at $P=100 P a$ have been reported. The most (a)

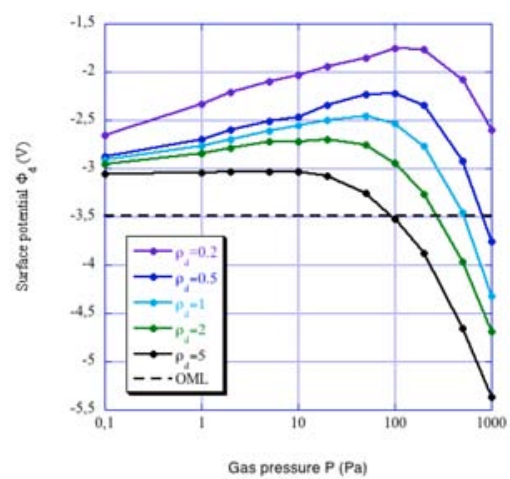

(b)

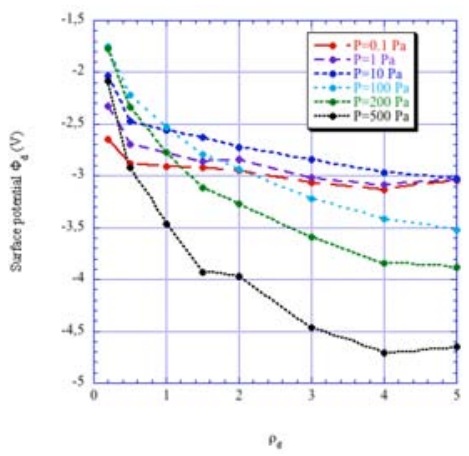

(c)

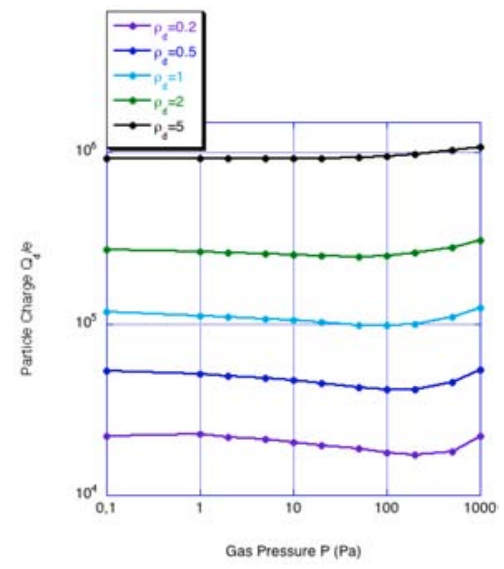

(d)

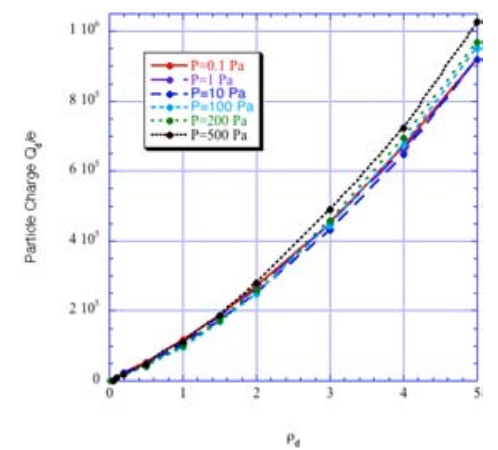

Fig. (4). Particle potential (a) as a function of the gas pressure for different particle size (dashed line is OML theory) and (b) as a function of particle radius for different gas pressure. Particle charge number (c) as a function of the gas pressure for different particle size and (d) as a function of particle radius for different gas pressure. 
(a)

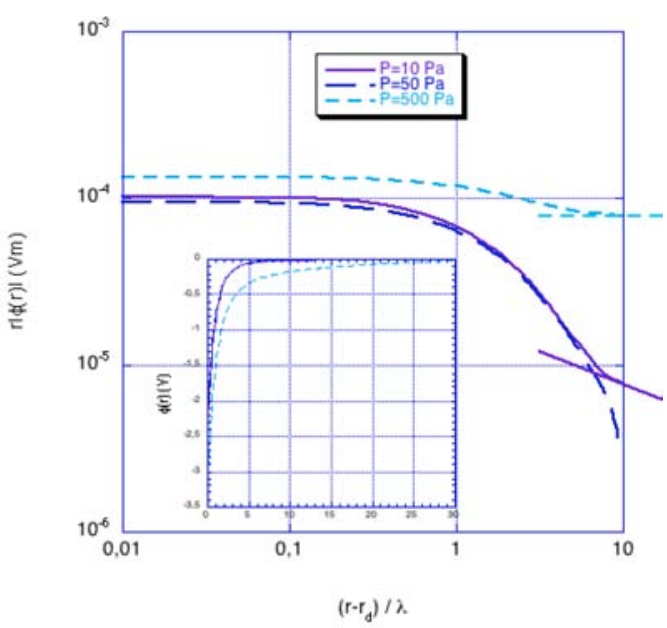

(b)

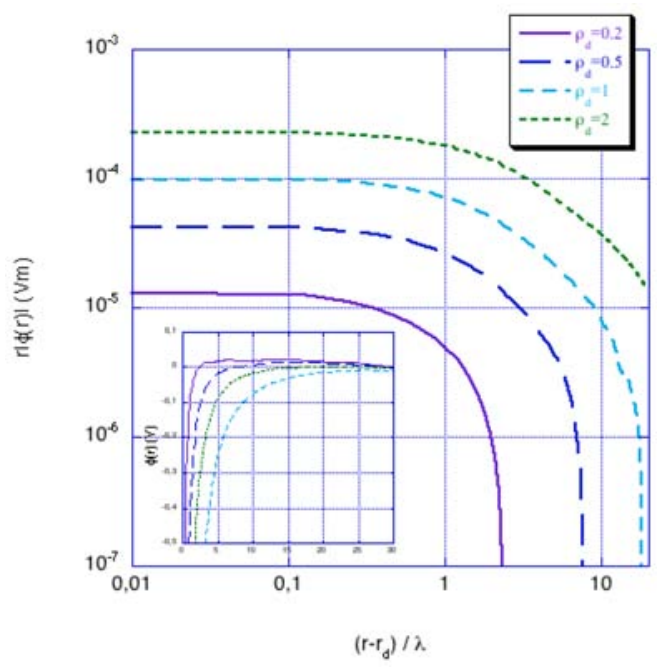

(c)

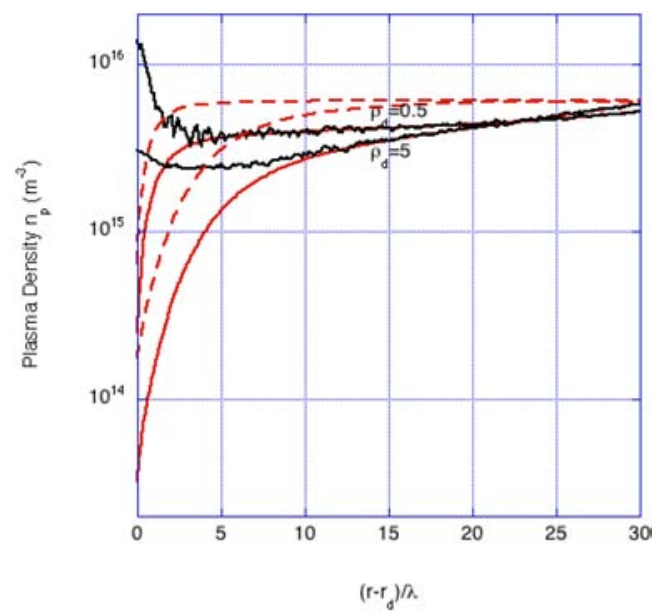

Fig. (5). Electric potential distribution (a) for a particle radius $\rho_{d}=1$ at different gas pressure and (b) for a gas pressure $P=100 \mathrm{~Pa}$ at particle radius. (c) Radial profiles of electron (red lines) and ion (black lines) density for two different particle size $\rho_{d}=0.5$ and $\rho_{d}=5$, both at $P=100 \mathrm{~Pa}$. The dashed lines represent the Boltzmann relation. evident feature is the rapidly increasing of ion density close to the particle due to the spherical geometry; the majority of ions orbits around the particle with a significant angular momentum and does not strike the dust. Differently from the planar sheath behaviour (monotonic decreasing density), where all the ions entering the sheath are continuously accelerated until reaching the surface. As a confirmation in the behavior of ion density for large particle radius; the ion rise decreases with the increasing of the particle size. As regards the electrons behavior, the discrepancy from the Boltzmann representation (dashed red lines) reaches a factor up to 5 close to the particle. Finally, as already shown in Fig. (5b), the sheath width grows from $\lambda_{D i}$ to a size of order of several $\lambda_{D e}$ with $\rho_{d}$.

\section{ADVANCED MODELS IN SHOCK-WAVES}

The first investigation in the Laboratory of Radiative Gas Dynamics dedicated to the calculation of nonequilibrium spectral intensity of radiation emitted by the shock-heated gases was performed and presented in 1997 [27]. Since then our group is involved in this area of scientific researches [28-30]. Two parts of activities are distinguished in this area. The first one is connected with the development and investigation of properties of hybrid radiative-collisional model. In our opinion hybrid and full radiative-collisional models differ in the following way. In the full radiativecollisional models all electronic excited states of particles presented in the gas mixture and processes relevant for the transitions among these states are taken into account. The problem with this approach is that there is limited amount of reliable data in the temperature region of interest regarding the rates (or cross sections) of processes accounted for in the model (due to the fact that there are a lot of processes generally because of the number of states included in the full model). Another problem is the extreme computational cost of these models [31]. Consequence of it is inability of inclusion of them into high fidelity Computational Fluid Dynamics (CFD) codes. However, in the hybrid radiativecollisonal models not all but only those of electronic excited states of particles (and processes responsible for transitions among them) are accounted for that are important from the point of view of radiation. It should significantly reduce cost of computations and uncertainty in the obtained results. The hybrid radiative-collisional model developed in our group consists of the following parts:

- Euler system of equations for the determination of parameters of the flow in the relaxation zone behind the shock front. The equation of energy is modified in order to take into account the fact that the considered gas mixture is multicomponent and chemically reacting:

$$
\begin{aligned}
& \frac{d}{d x}(\rho u)=0 ; \quad \frac{d}{d x}\left(p+\rho u^{2}\right)=0 ; \quad \frac{d}{d x}\left(h+\frac{u^{2}}{2}\right)=0 \\
& p=\frac{\rho R_{0} T}{M_{\Sigma}} ; \quad M_{\Sigma}=\sum_{i=1}^{N_{s}} \mu_{i} x_{i} ; \quad \mu_{i}=m_{i} N_{A}
\end{aligned}
$$


$h=\left[\left(T-T^{0}\right)\left(\frac{5}{2}+\sum_{i=1}^{N_{M}} x_{i}\right)+\sum_{i=1}^{N_{M}} x_{i} \sum_{j=1}^{N_{V, i}} g_{i, j} \frac{\theta_{i, j}}{\exp \left(\theta_{i, j} / T_{V, i, j}\right)-1}\right]$

Here $p, \rho, u, T$ - pressure, density, velocity and translational temperature; $x_{i}, m_{i}$ - molar fraction and mass of $i$-th chemical component (atom or molecule); $T_{V, i, j}, \theta_{i, j}, g_{i, j}$ - vibrational temperature, characteristic vibrational temperature and degeneracy of $j$-th vibrational mode of $i$-th molecule, $N_{s}$ - number of chemically reacting components; $N_{M}$ - number of diatomic and triatomic molecules; $N_{V, i}$ - number of vibrational modes of $i$-th molecule.

The system of chemical kinetic equations:

$\frac{d X_{k}}{d x}=\sum_{j=1}^{N_{r}}\left[\left(b_{k j}-a_{k j}\right) k_{j}^{f} \prod_{i=1}^{N_{s}} X_{i}^{a_{i j}}+\left(a_{k j}-b_{k j}\right) k_{j}^{r} \prod_{i=1}^{N_{s}} X_{i}^{b i j}\right]$,

$k=1, \ldots, N_{s}$

Here $X_{k}$ - molar volume concentration, $a_{k j}, b_{k j}$ stoichiometric coefficients of the $j$-th forward and backward reactions for the $k$-th chemical component, $k_{j}^{f}, k_{j}^{r}$ - forward and backward rate coefficient of $j$-th reaction. The system of chemical equations can be written in the following form:

$\sum_{i=1}^{N_{s}} a_{i j}\left[X_{i}\right] \stackrel{k_{j}^{f}}{=} \sum_{i=1}^{N_{s}^{r}} b_{i j}\left[X_{i}\right]$

Here $\left[X_{i}\right]$ - symbol that correspond to the $i$-th chemical component, $N_{r}$ - total number of reactions. It is worth noticing that for the chosen electronic excited states of molecules the relevant set of chemical equations formulated and kinetic equations are solved in order to determine populations of these states.

Equations for the determination of temperature of vibrational modes of molecules:

$\frac{d e_{m}}{d t}=Q_{V T}^{m}+Q_{C V}^{m}$

The relaxation times are usually estimated using the Millikan-White approximations combined with Park correction. The mutual influence of chemical kinetic and vibrational relaxation is taken into account in the model (CVDV interaction).

Equation for the determination of temperature of electron gas:

$\frac{d}{d x}\left(\frac{3}{2} T_{e} X_{e} u\right)+T_{e} X_{e} \frac{d u}{d x}=Q_{e i}+Q_{e a}+Q_{a i}+Q_{i o n}+Q_{e v}$
The processes responsible for the heating/cooling of the electron gas are as follows: elastic electron-ion and electron-atom collisions; associative ionization/ dissociative recombination; ionization; interaction of electron gas and vibrational modes of molecules.

- The just-overlapping line model is used for the spectral intensity of radiation calculations. The equations of the model are as follows:

$$
\begin{aligned}
& j_{\lambda}=3.202 \cdot 10^{-10} \frac{N_{e e l}}{Q_{V R} \lambda^{6}} \sum_{V}^{\prime} \sum_{V}^{\prime \prime} \frac{S_{V^{\prime} V^{\prime \prime}}}{\left|\Delta B_{V}\right|} \exp \left[-\frac{h c}{k T_{V}} E_{e e l}\left(V^{\prime}\right)\right] \\
& \exp \left[-\frac{h c}{k T_{R}} \frac{B_{V^{\prime}}}{\Delta B_{V}}\left(\omega-\omega_{V^{\prime} V^{\prime \prime}}+B_{V^{\prime}}\right)\right]
\end{aligned}
$$

The most important value here is $S_{V^{\prime} V^{\prime \prime}}$ which is the strength of vibronic transition. It is connected with the square of moment of vibronic transition in accordance with the following relation:

$$
\begin{aligned}
& S_{V^{\prime} V^{\prime \prime}}=\left(2-\delta_{0, \Lambda^{\prime}+\Lambda^{\prime \prime}}\right)(2 S+1)\left|R_{V^{\prime} V^{\prime \prime}}\right|^{2} \\
& \left|R_{V^{\prime} V^{\prime \prime}}\right|^{2}=\frac{3 h}{64 \pi^{4} a_{0}^{2} e^{2}} \frac{2-\delta_{0, \Lambda^{\prime}}}{2-\delta_{0, \Lambda^{\prime}+\Lambda^{\prime \prime}}} \frac{1}{\omega_{V^{\prime} V^{\prime \prime}}^{3}} \cdot A_{V^{\prime} V^{\prime \prime}}
\end{aligned}
$$

Here $A_{V^{\prime} V^{\prime \prime}}$ is the Einstein coefficient of vibronic transition. One can obtain the $A_{V^{\prime} V^{\prime \prime}}$ as a result of solution of quantum mechanical problem of the calculation of vibronic wave functions.

The full description of the model can be found in [28-30]. It is worth noting that what is presented here is actually a framework. Using it different gas mixtures and chemical kinetics models can be studied. Using the method it is possible to study nonequilibrium and equilibrium spectral intensities of radiation emitted by shock-heated gases. The second part of activity in our group is dedicated to the systematic processing of experimental data obtained on different shock tube facilities all around the world by means of the model developed in our group. Up to the moment we have performed the analysis of results obtained on the shock tubes of NASA Ames Research Center (EAST facility) [32,33], Chofu Aerospace Center [34], Queensland University (X2 facility) [35], Institute of Mechanics MSU [36]. By means of our model we have studied spectral intensity of radiation of shock-heated $\mathrm{N}_{2}-\mathrm{O}_{2}, \mathrm{CO}_{2}-\mathrm{N}_{2}$, $\mathrm{CH}_{4}-\mathrm{N}_{2}$ gas mixtures. Examples of comparison of numerical and experimental results are presented in Figs. $(6,7)$.

\section{SIMULATION OF HYPERSONIC RAREFIED FLOWS WITH THE IMMERSED-BOUNDARY METHOD}

\subsection{Introduction}

Computational Fluid Dynamics (CFD) studies of realworld industrial applications has typically to deal with complex geometries. A very time-consuming step in these cases is represented by the generation of the body-fitted computational mesh. A promising alternative is the Immersed Boundary (IB) method. In this method a Cartesian 
grid is employed and the effect of the solid boundary is simulated by suitable boundary conditions. The method has been first applied to incompressible flows [37-41] and later extended to preconditioned compressible Navier-Stokes (NS) equations [42]. Local mesh refinement techniques have been implemented in order to resolve the strong gradients that typically appear in boundary layers and shock waves $[43,44]$.

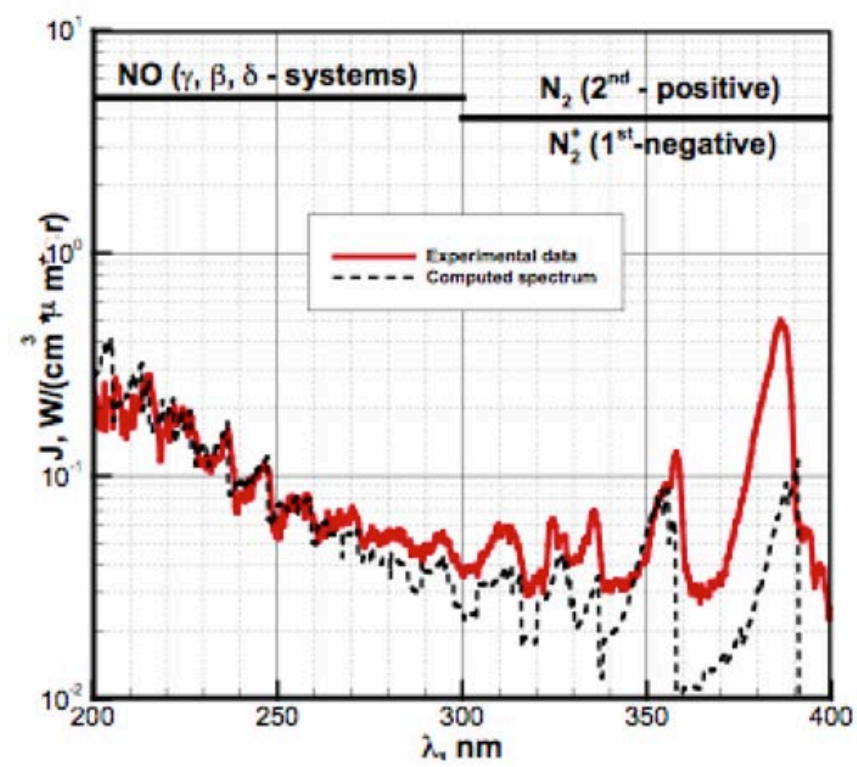

Fig. (6). Nonequilibrium spectral emissivity of the relaxation zone behind the shock wave initiated in $80 \% \mathrm{~N}_{2}-20 \% \mathrm{O}_{2}$ gas mixtures at $P=1$ Torr,$V_{s h}=5.56 \mathrm{~km} / \mathrm{s}$.

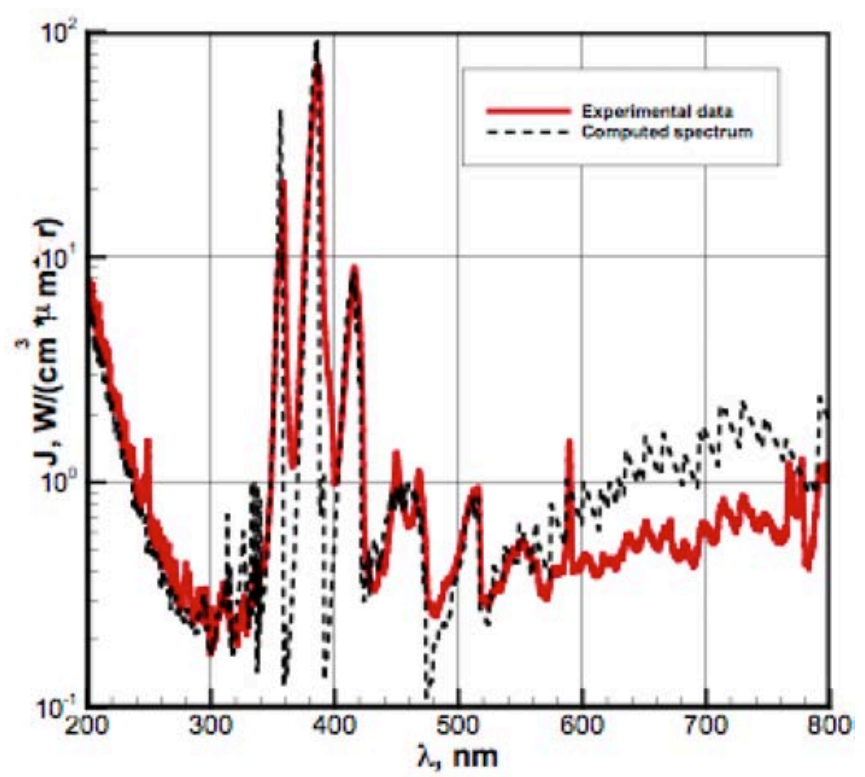

Fig. (7). Nonequilibrium spectral emissivity of the relaxation zone behind the shock wave initiated in $30 \% \mathrm{~N}_{2}-70 \% \mathrm{CO}_{2}$ gas mixtures at $P=0.3$ Torr,$V_{s h}=6.76 \mathrm{~km} / \mathrm{s}$.

In this work, the IB method is extended to the solution of flows with rarefaction effects and is validated against DSMC simulations of a shock wave-boundary layer interaction.

\subsection{Immersed-Boundary Navier-Stokes (IBNS) Equation Solver}

The preconditioned Navier-Stokes (NS) equations for a steady laminar flow of a perfect gas are solved [43]. The Prandtl number is equal to 0.71 and a temperature exponent for the viscosity coefficient equal to 0.75 has been employed.

A pseudo-time derivative is added to the NS equations in order to use a time marching approach to compute the steadystate solution. The preconditioning matrix proposed in [45] is used to pre-multiply such a pseudo-time derivative to enhance the efficiency of the solution at all values of the Mach number. The equations are discretized by an implicit Euler scheme in the pseudo-time. The space discretization is based on a cell-centred finite volume approach. The convective terms are discretized using a second-order-accurate upwind flux-difference-splitting scheme. The viscous terms are discretized by second-orderaccurate centred differences. The resulting discrete system is solved direction-by-direction using a BiCGStab [46] approach, the boundary conditions being treated explicitly (see [43] for details).

An efficient local grid refinement technique is employed for clustering cells near the immersed boundary and at other high-flow-gradient regions. For each face, the contributions of the neighbor cells are collected to build the corresponding convective and diffusive operators for the cell, the maximum number of neighbors being limited to two for the present two-dimensional computations [43].

The IB technique used in this work is based on that proposed in $[39,40]$. In a preliminary step, the geometry under consideration, which is described by a closed polygon in two dimensions (a closed surface in three dimensions), is overlapped onto a Cartesian grid. Using the ray tracing technique based on the geometrical algorithms reported in [47], the computational cells occupied entirely by the flow are tagged as fluid cells; those whose centres lie within the immersed body are tagged as solid cells. Furthermore, the fluid cells neighboring solid ones are tagged as interface fluid cells, and the solid cells neighboring fluid ones are tagged as interface solid cells. Interface cells are used to enforce the boundary conditions between the solid body and the fluid.

In order to resolve strong gradients the computational mesh can be locally refined by an automated algorithm up to a user-specified resolution [48]. Note that the mesh refinement does not need to be isotropic. A parallel implementation has been obtained via domain decomposition and coded with the MPI protocol and computational load balancing is achieved by employing the METIS software [49].

Boundary conditions at solid surfaces are obtained by imposing flow velocity and temperature at the solid surface and then using a linear interpolation to calculate flow variables in the interface cells [43].

First-order slip velocity conditions [50] are employed.

\subsection{DSMC Method}

IBNS results have been compared to DSMC simulations. The latter use a standard DSMC implementation [51]. In the present case, a model with continuous internal energy is 
used. Energy in collision is distributed according to the Larsen-Borgnakke scheme with unitary collision relaxation number in order to simulate the constant- $\gamma$ behavior. The code implements a two-dimensional geometry with a bultiblock, stretched grid approach. Parallel computations, based on a domain decomposition approach and coded with MPI standard have been performed for the hypersonic ramp flow on a 16-cpu Linux cluster.

\subsection{Slip Model Validation}

As a first step, the slip flow model has been validated in a simple configuration. The gas is a Hard Sphere gas with the following properties:

$\mathrm{m}=28.9641 \mathrm{amu}$

$\gamma=1.4$

$\eta=5.6853 \cdot 10^{-6} \sqrt{\frac{T}{80}} P a \cdot s$

$\operatorname{Pr}=0.71$

The domain is a two-dimensional channel, $4 \cdot 10^{-3} \mathrm{~m}$ wide and periodic in the other direction. The walls are perfectly diffusing with wall temperature set to $150 \mathrm{~K}$. The average density in the channel is $n=9.13 \cdot 10^{21} \mathrm{~m}^{-3}$. The gas is subject to a volume acceleration in the longitudinal direction equal to $a_{x}=5 \cdot 10^{6} \mathrm{~m} / \mathrm{s}^{2}$.

The Knudsen number, based on the average density and the channel half-length, is $K n=0.05$. The local Knudsen number, instead, based on the local density and on the velocity gradient can reach the value 0.9 near the wall. The flow is highly rarefied, in fact.

For the NS simulations, uniform grids with 32 and 64 mesh points in the normal direction to the boundary provide the same results within plotting accuracy. Computations have been performed in a two-dimensional domain with periodic boundary-conditions in the streamwise direction, slip-velocity boundary conditions at the bottom wall, and symmetry conditions at the top boundary corresponding to mid-channel height.

DSMC simulations have been performed on a simulation domain $8 \cdot 10^{-3} \mathrm{~m}$ long with periodic boundary conditions in the streamwise direction and $2 \cdot 10^{-3} \mathrm{~m}$ wide with a diffuse wall at $y=0$ and symmetry condition at the upper limit. The simulation space has been discretized in 256x64 square cells $3.125 \cdot 10^{-5} \mathrm{~m}$ in length. The time step is $d t=5 \cdot 10^{-9} \mathrm{~s}$ and the number of simulated particles is about $3.25 \cdot 10^{5}$. Time averaging has been performed in order to decrease the statistical error.

The velocity profiles obtained by DSMC and IBNS with and without slip are reported in Fig. (8). In this plot, the position in the channel is divided by the channel half-length and the flow speed is divided by the maximum value at the center of the channel. The first-order slip model therefore allows to accurately reproduce DSMC results in this case even in conditions of strong rarefaction.

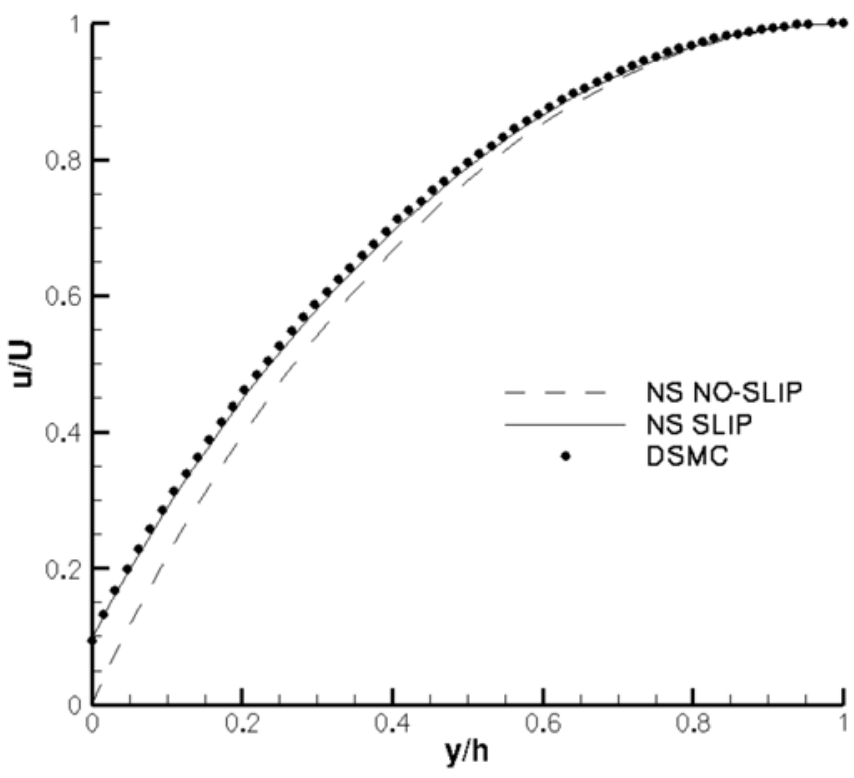

Fig. (8). Channel flow: velocity profiles.

\subsection{Hypersonic Ramp Flow}

The IBNS method has then been applied to the study of shock wave-boundary layer interaction as it appears in the supersonic flow over a wedge [52]. This conFiguration, representative of flows past deflection flaps, also features separation and re-attachment effects. Geometry and flow conditions are those studied in [52], the wedge angle being $35{ }^{\circ} \mathrm{C}$. The gas is Nitrogen. Due to the low temperatures involved, only rotational degrees of freedom are considered so the specific heat ratio $\gamma$ is 14; Prandtl number is set to 0.71 and the viscosity coefficient is assumed to have a temperature exponent of 0.75 .

Free-stream conditions are: $\rho_{\infty}=1.401 \times 10^{-4} \mathrm{~kg} / \mathrm{m}^{3}$, $V_{\infty}=1521 \mathrm{~m} / \mathrm{s}, T_{\infty}=9.06 \mathrm{~K}$. The corresponding freestream Mach and Reynolds number are 24.8 and 12,020, respectively. The wall is perfectly diffusing with wall temperature equal to $403.2 \mathrm{~K}$.

2D geometry is studied, the computational mesh being locally refined at the leading edge of the plate and in the recirculation region. A total of 80,000 cells have been used, the width of the smallest cell along the wall being $0.02 \mathrm{~mm}$. Steady-state calculations have been performed on a 16-core Linux cluster (8-core Intel Xeon @2.80Ghz processors) and about 20 minutes wall-clock time are required to obtain the solution (including the grid generation process).

DSMC simulations used 16 cpus on a Linux cluster. The mesh is made of 5 blocks with 1.7 million cells. The timestep is $d t=10^{-8} \mathrm{~s}$ and the number of simulated particles is 22 millions.

Fig. (9) shows the pressure and skin friction coefficients as obtained by DSMC and by IBNS method with slip conditions. Moss's results [52] are also reported for comparison. The IBNS results have been smoothed to avoid the typical small oscillations of the data at wall due to the linear reconstruction near the boundary. Concerning the 
DSMC results, a small residual statistical scatter is still visible after the time averaging procedure.

At the leading edge, the strong velocity gradient that induces nonlinear transport terms in the stress tensor is the likely cause of discrepancy between the particle methods on one side and the Navier-Stokes equation solver on the other.

Apart from this, the results compare favorably. A better agreement is found when using the slip conditions than when the standard no-slip conditions are considered (not shown in the figures). The skin friction plot shows that the separation zone is overestimated by the IBNS method. As a result, the flow velocity following re-attachment is underestimated. One possible reason for the discrepancy might be the use of slip coefficients for the Hard Sphere gas. Although, on general grounds, first-order slip coefficients are considered insensitive to the gas model [50], in the present case the position and the extent of the separation zone are found to be very sensitive to the slip coefficients. Further investigations are in progress in order to clarify this point.

Figs. (10, 11) provide the non-dimensional density contours, $\rho / \rho_{\infty}$, obtained by the IBNS and DSMC methods, respectively. The peak value of density is found about in the same region and is equal to 12.4 for the IBNS

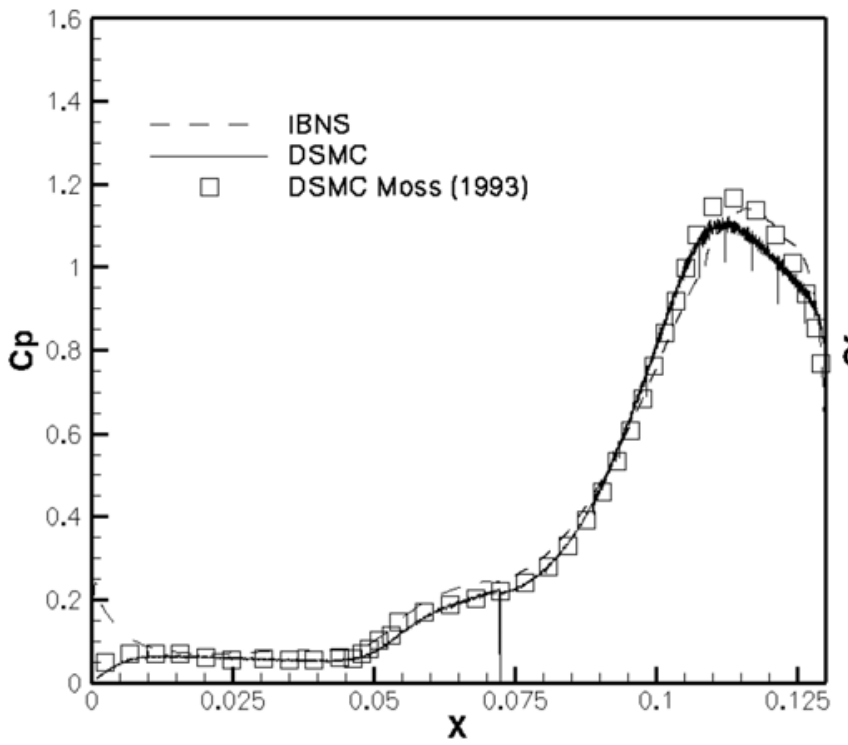

approach and to 14.3 for the DSMC, whereas the value reported in [52] is 14.8. Finally, Figs. $(12,13)$ report the streamlines (superposed to the density contours) obtained by the IBNS and DSMC methods, respectively. The agreement is satisfactory both qualitatively and quantitatively apart from the small discrepancy in the separation zone already discussed.

\subsection{Conclusion}

The results shown validate the Immersed Boundary method as an efficient approach for CFD studies of hypersonic rarefied gas flows. First-order slip-velocity boundary conditions have been tested that allow to account for rarefied gas effects. Automated, adaptive mesh refinement algorithms overcome the limits inherent in Cartesian grid approaches and a parallel implementation allows to tackle very demanding flow configurations.

Comparison with the results obtained from DSMC simulations is satisfactory. Discrepancies on the extension of the separation bubble call for further investigations.

\section{CONCLUSION}

A number of studies are reported that show the importance of kinetic simulation tools, in particular Monte

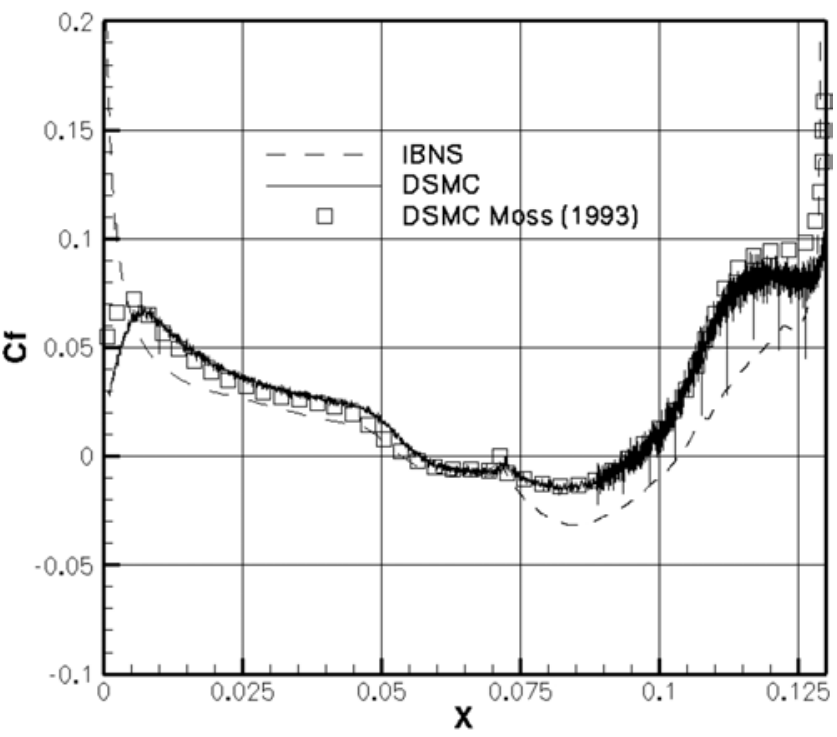

Fig. (9). Hypersonic ramp flow: pressure coefficient (left); skin-friction coefficient (right).

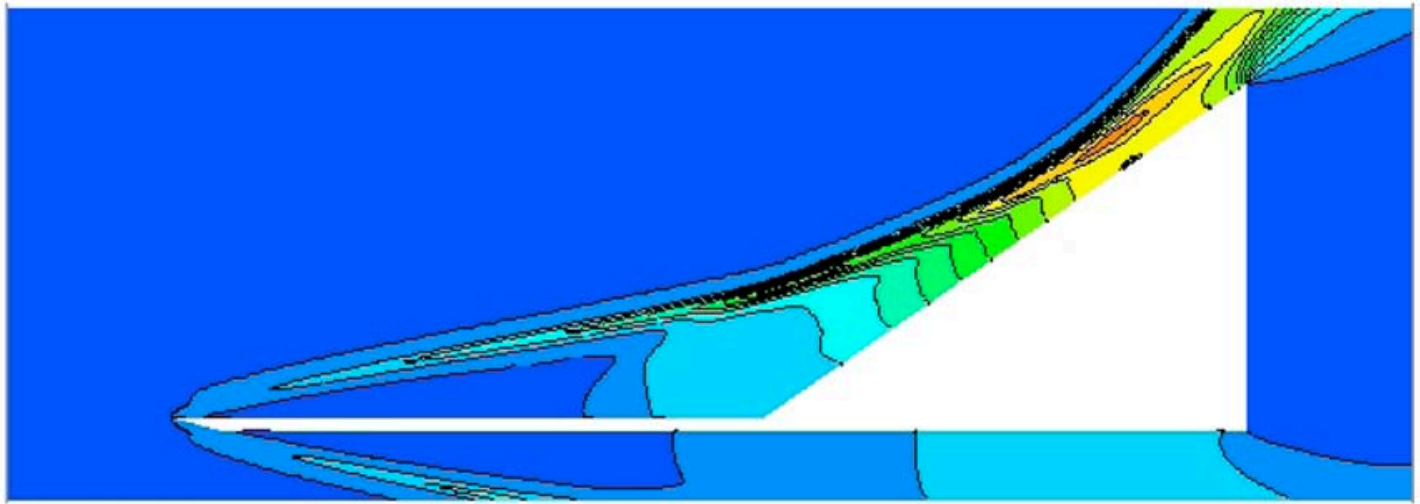

Fig. (10). Hypersonic ramp flow; Navier-Stokes equations: density contours $\left(\rho / \rho_{\infty} ; \Delta=1\right)$. 


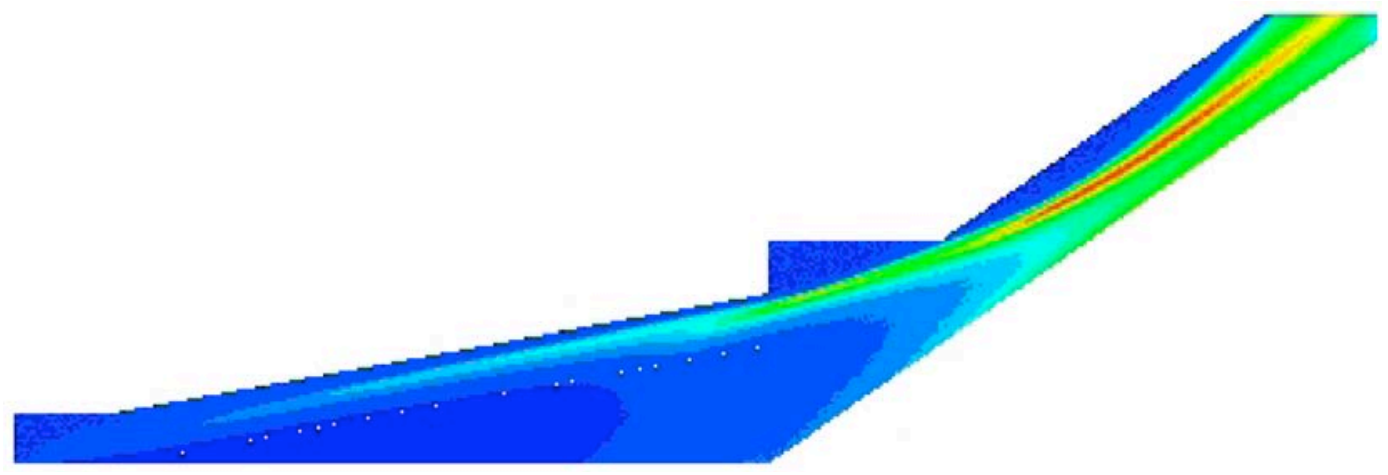

Fig. (11). Hypersonic ramp flow; DSMC: density contours $\left(\rho / \rho_{\infty} ; \Delta=1\right)$.

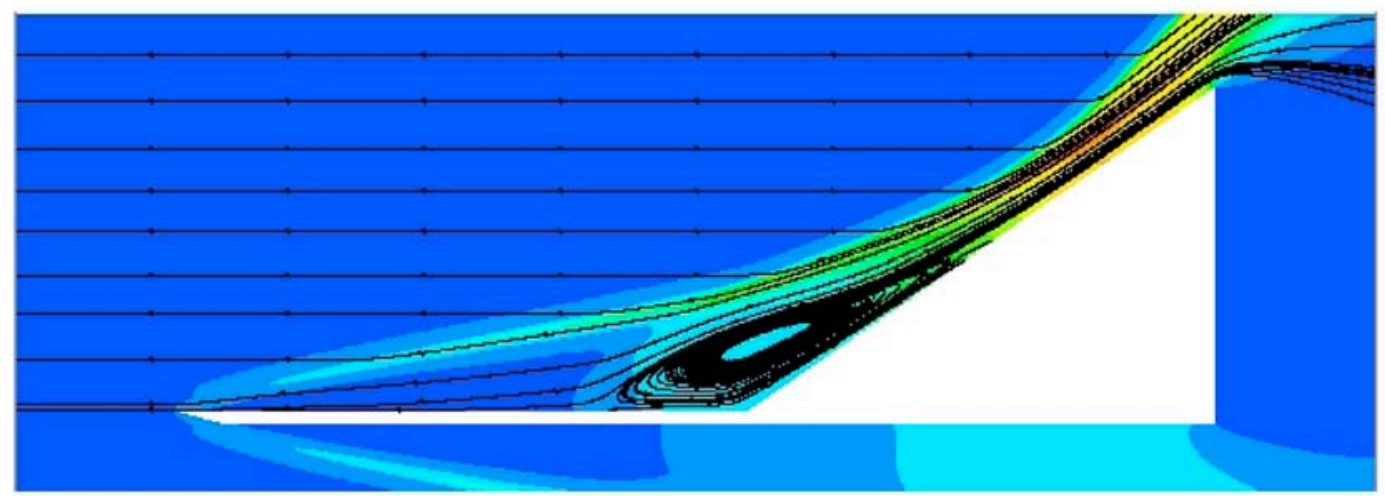

Fig. (12). Hypersonic ramp flow; Navier-Stokes equations: streamlines.

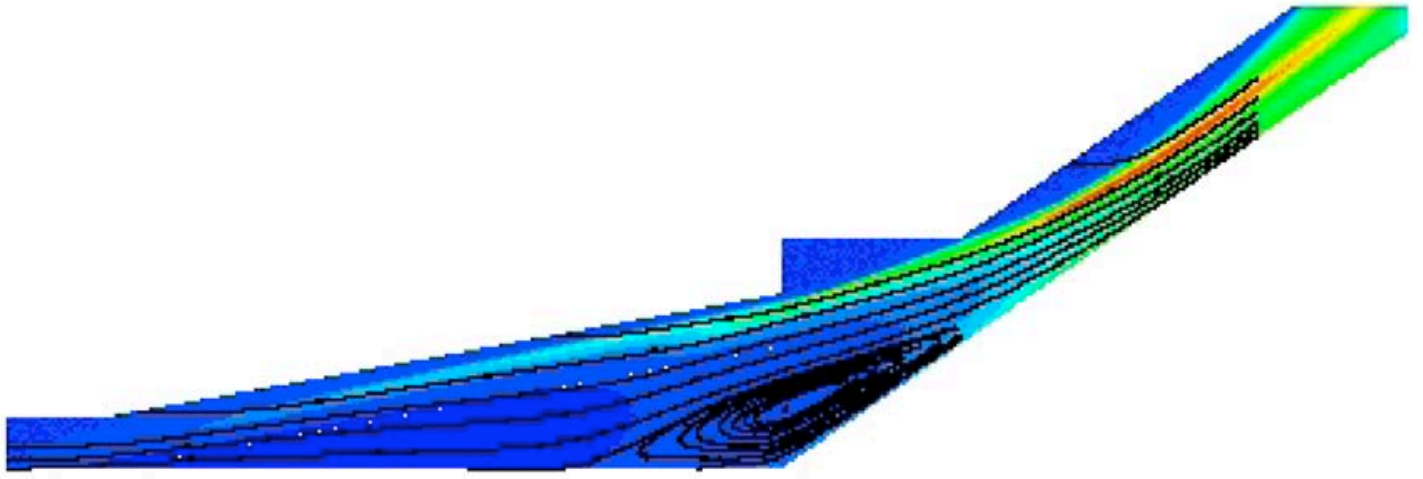

Fig. (13). Hypersonic ramp flow; DSMC: streamlines.

Carlo methods, for the description of nonequilibrium effects in hypersonic flows. Mass diffusion in steep shock wave fronts can therefore be analysed, which is not possible in the frame of a Navier-Stokes description of the fluid mixture. Plasma kinetic effects, arising in the interaction of macroscopic objects within a plasma environment, have been studied with a PIC technique. Consideration of thermal nonequilibrium effects also enters the modelling of nonequilibrium radiation from shock heated gas and a kinetic level collisional-radiative model has been presented that is Finally, gas rarefaction effects have been studied with a DSMC simulation tool and the results used to build a IB method for CFD solvers that takes these effects correctly into account. In addition, these results demonstrate that based on the results of such accurate kinetic models, significant progress has been achieved in devising nonequilibrium models for CFD solvers.

\section{CONFLICT OF INTEREST}

The authors confirm that this article content has no conflict of interest.

\section{ACKNOWLEDGMENTS}

The research leading to these results has received funding from the European Community's Seventh Framework Programme (FP7/2007--2013) under grant agreement $n$ 242311.

\section{REFERENCES}

[1] Longo S, Diomede P. Monte Carlo modeling of gas phase ion transport under thermal gradients and external fields. Eur Phys J Appl Phys 2004; 26(3): 177.

[2] Macrossan MN. A particle simulation method for the BGK equation, In: Proceedings of the 22nd International Symposium on Rarefied Gas Dynamics. AIP Conf Proc 2001; 585: 426-33. 
[3] Bruno D, Longo S. Monte Carlo simulation of nearly kinematic shock fronts in rarefied gases. Eur Phys J Appl Phys 2002; 17(3): 233-41.

[4] Bruno D, Longo SM. Capitelli A. BGK/MC method for the simulation of shock waves in binary mixtures. AIAA paper 2002-2895.

[5] Spanier J, Gelbard EM. Monte Carlo principles and neutron transport problems. Addison Wesley: USA 1969.

[6] Dupree SA, Fraley SK. A Monte Carlo primer: practical approach to radiation transport. Kluwer: Netherlands 2002.

[7] Lieberman MA, Lichtenberg AJ. Principles of plasmas discharges and material processing, Wiley: USA 2005.

[8] Bird GA. Molecular gas dynamics and the direct simulation of gas flows. Clarendon: Oxford 1994

[9] Longo S, Diomede P. A Monte Carlo model for seeded atomic flows in the transition regime. J Comput Phys 2009; 228(10): 3851-7.

[10] Kolobov VI, Arslanbekov RR, Aristov VV, Frolova AA. Unified solver for rarefied and continuum flows with adaptive mesh and algorithm refinement. J Comput Phys 2007; 223(2): 589-608.

[11] Zel'dovich YB, Raizer YP. Physics of shock waves and hightemperature hydrodynamic phenomena. Dover: New York 2002.

[12] Fortov VE, Ivlev AV, Khrapak SA, Khrapak AG, Morfill GE. Complex (dusty) plasmas: Current status, open issues, perspectives. Phys Rep 2005; 421: 1-103.

[13] Ishihara O. Complex plasma: dusts in plasma. J Phys D 2007; 40(8): R121-47.

[14] Shukla PK, Eliasson B. Fundamentals of dust-plasma interactions. Rev Mod Phys 2009; 81: 25-44.

[15] Khrapak SA, Morfill GE. Basic processes in complex (dusty) plasmas: Charging, interactions, and ion drag force. Contrib Plasma Phys 2009; 49 (3): 148-68.

[16] Allen JE. Probe theory - the orbital motion approach. Phys Scrip 1992; 45: 497-503.

[17] Lampe M. Limits of validity for orbital-motion-limited theory for a small floating collector. J Plasma Phys 2001; 65(3): 171-80.

[18] Tskhakaya D, Matyash K, Schneider R, Taccogna F. The Particle-inCell method. Contrib Plasma Phys 2007; 47(8-9): 549-94.

[19] Taccogna F, Longo S, Capitelli M. A particle-in-cell/Monte Carlo model of the $\mathrm{Ar}^{+}$ion collection in $\mathrm{He}$ gas by a cylindrical langmuir probe in the transition regime. Eur Phys J Appl Phys 2003; 22: 29-39.

[20] Taccogna F, Longo S, Capitelli M. PIC model of the ion collection by a Langmuir probe. Contrib Plasma Phys 2004; 44(7-8): 594-600.

[21] Taccogna F, Longo S, Capitelli M. Ion orbits in a cylindrical Langmuir probe. Phys Plasma 2006; 13: 043501.

[22] Taccogna F, Longo S, Capitelli M. Ion-neutral collision effects in Langmuir probe theory. Contrib Plasma Phys 2008; 48(5-7): 509-14.

[23] Taccogna F. Dust in plasma I. Particle size and ion-neutral collision effects. Contrib Plasma Phys 2012; 52(9): 744-55.

[24] Verboncoeur JP. Symmetric spline weighting for charge and current density in particle simulation. J Comput Phys 2001; 174: 421-7.

[25] Press WH, Teukolsky SA, Vetterling WT, Flannery BP. Numerical recipes in fortran 77: The Art of scientific computing. New York: Cambridge University Press 2001

[26] Nanbu K, Kitatani Y. An ion-neutral species collision model for particle simulation of glow discharge. J Phys D 1995; 28(2): 324-30.

[27] Kuznetsova LA, Surzhikov ST. Spectral radiation of shock waves and radiative models of diatomic molecules. AIAA paper 97-2564.

[28] Kudryavtsev NN, Kuznetsova LA, Surzhikov ST. Kinetics and nonequilibrium radiation of $\mathrm{CO}_{2}-\mathrm{N}_{2}$ shock waves. AIAA paper 2001-2728.

[29] Surzhikov ST, Sharikov I, Capitelli M, Colonna G. Kinetic models of non-equilibrium radiation of strong air shock waves. AIAA paper 2006-0586.

[30] Dikalyuk AS, Surzhikov ST, Shatalov OP, Kozlov PV, Romanenko YV. Nonequilibrium radiation behind the strong shock waves in martian and titan atmospheres: Numerical rebuilding of experimental data. AIAA paper 2012-0795.
[31] Panesi M, Magin T, Bourdon A, Bultel A, Chazot O. Fire II flight experiment analysis by means of a collisional-radiative model. J Thermophys Heat $\operatorname{Tr} 2009$; 2(2): 236-48

[32] Grinstead JH, Wilder MC, Wright MJ, et al. Shock radiation measurements for mars aerocapture radiative heating analysis. AIAA paper 2008-1272.

[33] Cruden BA, Prabhu D, Martinez R, Le H, Bose D, Grinstead JH Absolute radiation measurement in venus and mars entry conditions. AIAA paper 2010-4508.

[34] Takayanagi H, Fujita K. Absolute radiation measurements behind strong shock wave in carbon dioxide flow for mars aerocapture missions. AIAA paper 2012-2744.

[35] Brandis AM, Morgan RG, McIntyre TJ, Jacobs PA. Nonequilibrium radiation intensity measurements in simulated titan atmospheres. J Thermophys Heat Tr 2010; 24(2): 291-300.

[36] Kozlov PV, Romanenko YV, Shatalov OP. Radiation intensity measurement in simulated Martian atmospheres on the double diaphragm shock tube. In: Proceedings of the 4th International Workshop on Radiation of High Temperature Gases in Atmospheric Entry, ESA SP-689. Lausanne: Swiss 2010.

[37] Peskin CS. Flow patterns around heart valves: A numerical method. J Comput Phys 1972; 10: 252.

[38] Mohd-Yusof J. Combined immersed boundaries/B-splines methods for simulations of flows in complex geometries. CTR Annual Research Briefs, NASA Ames - Stanford University 1997.

[39] Fadlun EA, Verzicco R, Orlandi P, Mohd-Yusof J. Combined immersed-boundary finite-di erence methods for three-dimensional complex flow simulations. J Comput Phys 2000; 161: 35 .

[40] Iaccarino G, Verzicco R. Immersed boundary technique for turbulent flow simulations. Appl Mech Rev 2003; 56: 331-47.

[41] Mittal R, Iaccarino G. Immersed boundary methods. Annu Rev Fluid Mech 2005; $37: 239$.

[42] De Palma P, de Tullio MD, Pascazio G, Napolitano M. An immersed boundary method for compressible viscous flows. Comput Fluids 2006; $35: 693$.

[43] de Tullio MD, De Palma P, Iaccarino G, Pascazio G, Napolitano M. An immersed boundary method for compressible flows using local grid refinement. J Comput Phys 2007 ; 225: 2098-117.

[44] de Tullio MD, De Palma P, Napolitano M, Pascazio G. Recent advances in the development of an immersed boundary method for industrial applications. In: Kuzmin A, Ed. Computational Fluid Dynamics 2010. Springer: Berlin, Heidelberg 2011; pp. 601-6.

[45] Merkle CL. Preconditioning methods for viscous flow calculations, In: Hafez M, Oshima K Eds. Computational Fluid Dynamics Review 1995. New York: Wiley 1995: p. 419.

[46] van der Vorst H. Bi-CGSTAB: a fast and smoothly converging variant of Bi-CG for the solution of non-symmetric linear systems. SIAM J Sci Stat Comput 1992; 13: 361

[47] J. O'Rourke, Computational Geometry in C. Cambridge University Press, Cambridge 1998.

[48] Kang S, Iaccarino G, Ham F. DNS of buoyancy-dominated turbulent flows on a bluff body using the immersed boundary method. J Comput Phys 2009; 228: 3189

[49] Karypis G, Kumar V. A fast and high quality multilevel scheme for partitioning irregular graphs. SIAM J Sci Comput 1998; 20: 359.

[50] Hadjiconstantinou NG. The limits of Navier-Stokes theory and kinetic extensions for describing small-scale gaseous hydrodynamics. Phys Fluids 2006; 18: 111301.

[51] Bruno D, Capitelli M, Esposito F, Longo S, Minelli P. Direct simulation of non-equilibrium kinetics under shock conditions in nitrogen. Chem Phys Lett 2002; 360: 31 .

[52] Moss JN, Rault DF, Price JM. Direct Monte Carlo simulations of hypersonic viscous interactions including separation, In: Shizgal BD, Weaver DP, Eds. Rarefied Gas Dynamics: Space Science and Engineering. Progress in Aeronautics and Aeronautics, AIAA Washington 1993; vol. 160: pp. 209-20. 